\title{
Selenium intake, status, and health: a complex relationship
}

\author{
Margaret P Rayman ${ }^{1}$ (D)
}

Received: 6 March 2019 / Accepted: 24 July 2019 / Published online: 6 August 2019

(C) The Author(s) 2019

\begin{abstract}
Both selenium (Se) deficiency and excess are found in natural locations throughout the world, though Se excess can also be caused by supplementation with Se. Both have been associated with adverse health effects that have often been characterized by a U-shaped relationship. Some health effects, such as increased mortality, are associated with both low and high Se status. Certain people and populations are better able to tolerate low or high Se intake than others; there are a number of possible explanations for this fact. Firstly, it may relate to the presence of polymorphisms (SNPs) in genes that improve the ability to deal with a low or high Se intake. Secondly, high Se status, with apparent absence of toxicity and even beneficial effects, can be found in populations exposed to toxic elements that are known to interact with Se, forming complexes in some cases. Thirdly, beneficial and harmful effects of Se depend on Se dose and form (speciation); for instance, at a high dose, selenomethionine (SeMet) has toxic effects that are mediated by metabolism to selenols/selenolates that can redox-cycle, generate superoxide radicals and react with thiols/diselenides to produce selenyl sulphides/disulphides. Finally, it is possible that exposure to a high Se intake from birth or from a very young age may alter the composition of the gut microbiota in such a way that excess Se is more readily excreted, thus reducing its toxicity.
\end{abstract}

Keywords Selenium · Deficiency · Excess · Adverse health effects · Toxic elements · Dose - Speciation · Polymorphisms · Microbiota

\section{Introduction}

The highly insightful observation made by Confucius (551 to $479 \mathrm{BC})$, "Excess and deficiency are equally at fault", is as relevant today as it was 2500 years ago, and it applies to almost all nutrients, though it is particularly apposite to selenium (Se).

Se intake is extremely variable across the world [1] owing to a number of factors, including the Se content of the soil in which crops and fodder are grown, Se speciation, soil $\mathrm{pH}$ and organic-matter content, and the presence of ions that can complex with Se [2]. This fact enables the effects of both Se deficiency and excess to be observed in the natural world.

Margaret P Rayman

m.rayman@surrey.ac.uk

1 Department of Nutritional Sciences, Faculty of Health and Medical Sciences, University of Surrey, Guildford GU2 7XH, UK

\section{Effects of Se deficiency}

Some adverse health conditions associated with Se deficiency are listed in Table 1.

A further example that is less well known relates to the reduction in risk of incident tuberculosis (TB) in HIVinfected subjects who were treated with Se. Because HIV is an immune-deficiency syndrome, those with this disease commonly suffer from other infections, such as TB, particularly as the condition progresses [15]. In a 24-month, fourarm, randomized, controlled trial (RCT) in 878 HIV-infected adults in Botswana, who were randomized to Se, multivitamins, Se+multivitamins or placebo, treatment with $200 \mu \mathrm{g}$ Se+multivitamins significantly reduced the risk of immune decline and morbidity, while multivitamins alone and $\mathrm{Se}$ alone had no effect [16]. Importantly, Se vs. placebo significantly reduced the risk of incident TB: hazard ratio (HR) 0.20, 95\%; confidence interval (CI): $0.04,0.95 ; P=0.043$. When the Se and Se+multivitamin arms were combined, the risk of TB was also reduced: HR 0.32, 95\% CI: 0.11, 0.93; 
Table 1 Conditions that have been associated with Se deficiency [3]

\begin{tabular}{ll}
\hline Condition & Reference \\
\hline Keshan disease & {$[2]$} \\
Kashin-Beck disease & {$[4]$} \\
Increased viral virulence & {$[5]$} \\
Increased mortality & {$[6]$} \\
Poorer immune function & {$[7]$} \\
Problematic fertility/reproduction & {$[3]$} \\
Thyroid autoimmune disease & {$[8]$} \\
Cognitive decline/dementia & {$[9-11]$} \\
Type 2 diabetes & {$[12]$} \\
Prostate cancer risk & {$[13]$} \\
Colorectal cancer risk in women (seen in EPIC) & {$[14]$} \\
\hline
\end{tabular}

$P=0.036$ [15]. This is an important finding in relation to HIV outcome, particularly in sub-Saharan Africa, where TB can often be fatal.

\section{Effects of Se excess}

The effects of Se excess are probably less well known. Apart from some occasional cases of overdose where people have ingested wrongly formulated supplements [17], Se excess has mostly been observed in RCTs, where doses of $200 \mu \mathrm{g} /$ day or more of Se have been given for a substantial period of time (see Table 2).

\section{Adverse health effects associated with, or caused by, both Se deficiency and excess: Se and mortality}

It is of note that some health conditions are associated with both Se deficiency and excess, i.e. increased mortality, type 2 diabetes and increased prostate cancer risk [6, 12, 13]. Increased mortality is seen at both low and high plasma Se concentrations. This was first demonstrated by the Guallar group at Johns Hopkins School of Public Health, Baltimore,

Table 2 Health risks significantly related to selenium excess

\begin{tabular}{ll} 
Health condition & Reference \\
\hline Selenosis (Se toxicity) & {$[2,17]$} \\
Alopecia (seen in SELECT) & {$[18]$} \\
Dermatitis (seen in SELECT) & {$[18]$} \\
Non-melanoma skin cancer (seen in NPC trial) & {$[19]$} \\
Increased mortality & {$[6][20]$} \\
Type 2 diabetes (seen in the NPC trial) & {$[21]$} \\
Increased prostate cancer risk (seen in SELECT) & {$[22]$}
\end{tabular}

USA, which examined data from the US Third National Health and Nutrition Examination Survey. Serum Se was measured in 13,887 adult participants who were then followed up for mortality for up to 12 years [6] and subsequently for a further 6 years [3]. Mortality showed a U-shaped association, reaching a minimum at a serum Se concentration of $135 \mu \mathrm{g} / \mathrm{L}$.

Increased mortality was seen at the highest dose level of an $\mathrm{RCT}$ of Se in the Danish PRECISE trial, a randomized, double-blinded, placebo-controlled, clinical trial with four groups [20]. Participants were 491 male and female volunteers aged 60-74 years who were randomly assigned to treatment with 100,200 or $300 \mu \mathrm{g} \mathrm{Se}$ /day as Se-enriched yeast or placebo yeast for 5 years from randomization in 1998-1999 and were followed up for mortality for a further 10 years. In an intention-to-treat analysis, the HR $(95 \% \mathrm{CI})$ for all-cause mortality comparing $300 \mu \mathrm{g} \mathrm{Se} /$ day with placebo was $1.62(0.66$, 3.96) after 5 years of treatment and $1.59(1.02,2.46)$ over the entire follow-up period [20]. The relationship between cumulative mortality from all causes over time by treatment group in the Danish PRECISE RCT is shown in Fig. 1. The results of this study warn that a 300- $\mu \mathrm{g} /$ day dose of Se (as Se yeast) taken for 5 years in a country with moderately low Se status can increase all-cause mortality 10 years later.

The relationship between Se intake/status and mortality is an excellent example of the U-shaped association between Se and health. Figure 2 illustrates that relationship, additionally including data referred to in Tables 1 and 2 [23].

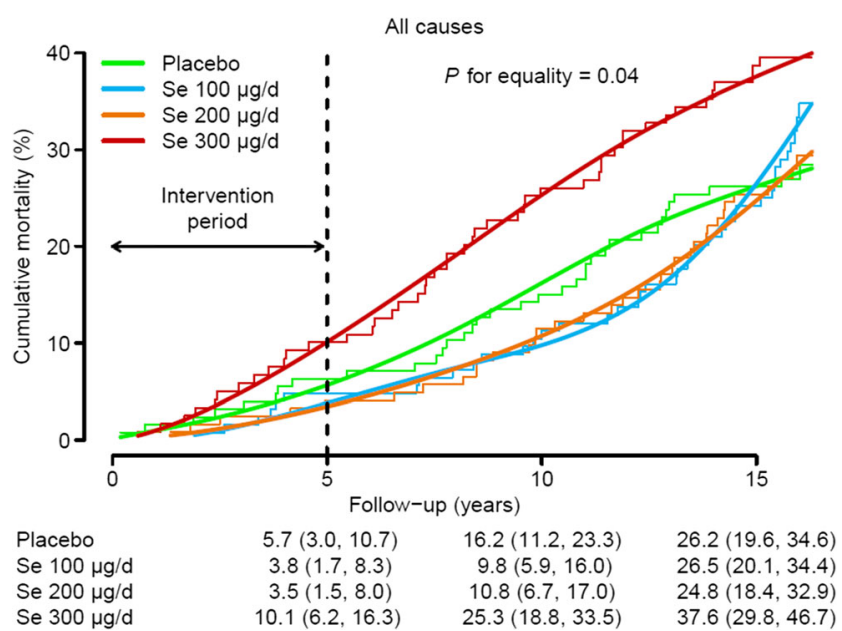

Fig. 1 Cumulative mortality from all causes over time by treatment group. Non-parametric cumulative mortality curves (step functions) were estimated via the Kaplan-Meier method and compared with results generated by the generalized Wilcoxon test. Parametric cumulative mortality curves (smooth lines) were estimated from spline-based parametric survival models with treatment-specific log cumulative hazards parameterized as natural cubic splines of log time with knots at the 33th and 67th percentiles of the uncensored log-time distribution. Cumulative mortality estimates ( $95 \%$ CIs) at 5, 10 and 15 years of follow-up by treatment group were obtained from spline-based parametric survival models. Se denotes selenium [20] (published with permission of FRBM) 
Fig. 2 U-shaped relationship between Se status and disease risk (for references, see [3])

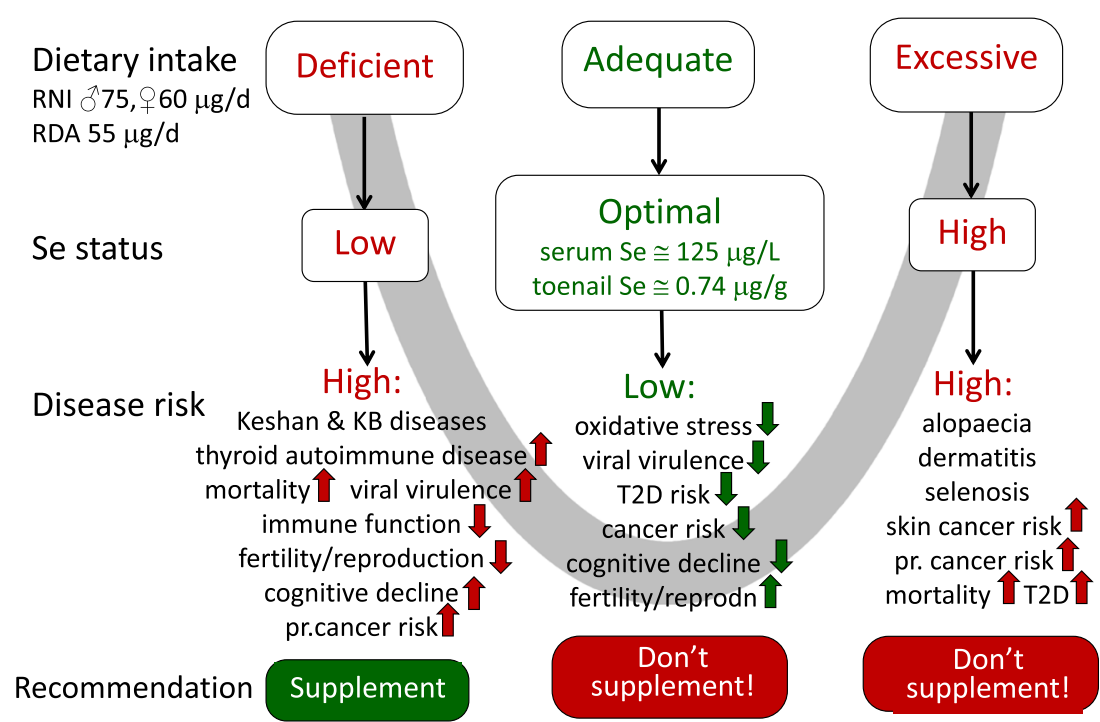

\section{Complexities in the relationship between Se intake and health effects: factors that need to be considered}

Though the results of the Danish PRECISE trial show excess mortality on long-term ( 5 year) exposure to $300 \mu \mathrm{g}$ Se/day as Se yeast of a population experiencing much lower Se exposure (plasma-Se concentration at baseline, $89 \mu \mathrm{g} / \mathrm{L}$, that rose to $284 \mu \mathrm{g} / \mathrm{L}$ after 5 years) [20], it is notable that population groups with long-term high-Se exposure appear to cope with a Se intake or status of the same magnitude as in that trial without suffering adverse effects, e.g. Inuit of Greenland and Northern Canada, populations of the Brazilian Amazon, and some local Chinese populations. There are a number of possible reasons for this, as outlined below.

1. It appears that some people and/or populations are better able to tolerate low or high Se intake than others owing to the presence of polymorphisms (SNPs) in genes that affect their ability to deal with a low or high Se intake. This has been best demonstrated in relation to deficient intake. The allele frequency of SNPs in several selenoprotein genes changes in response to differences in environmental Se [24], e.g. SNPs in:

(i) Selenoprotein genes, GPX1, DIO2, DIO3, SELENOS, SELENOM

(ii) Genes involved in $\mathrm{Se} / \mathrm{Sec}$ regulation of CELF1, SPS2, SEPSECS, ELAVL1, Ser-tRNA ${ }^{\text {Sec }}$ and SELENOP

(iii) Genes involved in excretion/detoxification of Se, such as INMT (indolethylamine $N$ methyltransferase).
In relation to the third point, Se is excreted by being metabolized to methylated Se products in breath or urine and to urinary selenosugars [25]. Individuals with the AA or AG INMT genotype can metabolize Se to trimethyl-selenonium ion (TMSe) in the urine, thereby excreting more Se and potentially reducing toxicity [26]. The frequency of TMSe production varies markedly between populations and individuals [26].

2. High Se status, with apparent absence of toxicity and even beneficial health effects, can be found in populations exposed to toxic elements, such as arsenic, cadmium and mercury, all of which are known to interact with Se. Sequestration in inert conjugates that can be excreted has been suggested as accounting for this. Various structures have been proposed for a complex between mercury and selenium or selenoprotein P (SELENOP), such as MeHg-Se-SELENOP [27] and (Hg-Se)n]m-SELENOP [28]. A complex of arsenic and Se [(GS)2AsSe] has been detected in rabbits [29] while a cadmium-Se complex with a molar ratio of $1: 1$ which was able to bind to SELENOP, has been shown to form in vitro [30].

(i) High mercury content is found in the diet of the Inuit in Greenland and Nunavik (Northern Quebec) as a result of consumption of top predator marine mammals and fish [31]. In a study of 852 Inuit adults who participated in the 2004 Nunavik Inuit Health Survey, up to $50 \%$ of plasma mercury was associated with SELENOP [32]. In the International Polar Year Inuit Health Survey (IHS) of Inuit in Canada conducted in 2007 and 2008, geometric mean blood Se and total $\mathrm{Hg}$ were $319.5 \mu \mathrm{g} / \mathrm{L}$ and $7.0 \mu \mathrm{g} / \mathrm{L}$, respectively [33]. High Se exposure was shown to lower the 
odds ratios for hypertension, stroke, and myocardial infarction associated with mercury exposure [33]. In the same study, participants with stroke had lower blood Se (geometric mean: $260 \mu \mathrm{g} / \mathrm{L}$ vs. $319 \mu \mathrm{g} / \mathrm{L}$ ) and dietary Se $(144 \mu \mathrm{g} /$ day vs. $190 \mu \mathrm{g} /$ day $)$ than individuals without stroke [34].

High levels of dietary mercury are also found in the Brazilian Amazon, notably in communities of the Tapajós River [mean intake (SD) $0.92(0.89) \mu \mathrm{g} / \mathrm{kg} /$ day] [35]. The dietary Se intake in these villages ranges from normal to very high owing to the consumption of large amounts of Brazil nuts, chicken, game and certain fish species [36]. In participants recruited from 12 communities along the Tapajós River, median blood and plasma Se were 228 (range 103-1500) $\mu \mathrm{g} / \mathrm{L}$ and 135 (range 54-913) $\mu \mathrm{g} / \mathrm{L}$, respectively [36]. Although blood and plasma concentrations exceeded concentrations considered toxic [blood Se: $1000 \mu \mathrm{g} / \mathrm{L}$ (US EPA 2002)], no signs of Se toxicity (dermal or breath) or sentinel symptoms (gastrointestinal disorders and motor/sensory deficits) were found, despite very high Se status in some individuals [36]. Interestingly, only three of 407 participants reported diabetes.

(ii) Rice easily takes up arsenic and elevated exposure is frequent in areas of Bangladesh, India, China and Thailand [37]. An antagonistic relationship between Se and arsenic was first observed in 1938 when it was discovered that Se-poisoned rats could be treated with arsenic [38]. Antagonism between arsenic and Se, whereby each reduces the toxicity of the other, has been well documented in animal models [39].

(iii) Cadmium, a potent pro-oxidant, accumulates in the kidney, increasing oxidative stress [40]; the demand for Se in the kidney may increase with higher cadmium exposure, as Se is required for the expression of the antioxidant enzyme, GPX3, which is produced in the kidney [41]. High cadmium was found in the Enshi area of China known for Se intake in the toxic range [42].

3. Beneficial and harmful effects of Se depend on Se dose and form (speciation) (see Fig. 3). It has been proposed that Se may have hermetic effects, i.e. a biphasic dose response to an agent characterized by a beneficial effect at a low dose and an inhibitory or toxic effect at a high dose [43]; this can be represented by the U-shaped response to increasing Se intake/status, as observed.

While selenoproteins are generally considered to have beneficial health effects, there are selenoproteins with paradoxical roles with potentially harmful outcomes [43], e.g. GPX1, implicated in insulin resistance [44] and SELENOP, associated with type 2 diabetes [45].

In relation to Se dose, as Se intake increases, particularly if the main source is selenomethionine (SeMet) or Se yeast (60\% of which is SeMet [46]), SeMet will increasingly replace methionine in body proteins, where its turnover is exceptionally slow (whole-body turn-over time, 363 days) [47]. Of course, SeMet can also be metabolized to selenide, whereby it can act as a source of selenoproteins. However, SeMet has toxic effects that are mediated by metabolism to selenols/selenolates that can redox-cycle, generate superoxide radicals, and react with thiols/diselenides to produce selenyl sulphides/ disulphides [20]. The latter can cause protein aggregation, inactivation of transcription factors, disruption of redoxregulated cell signaling, and endoplasmic reticulum (ER) stress [20]. It should be noted that Se, as sodium selenite, would be metabolized to hydrogen selenide, used for the

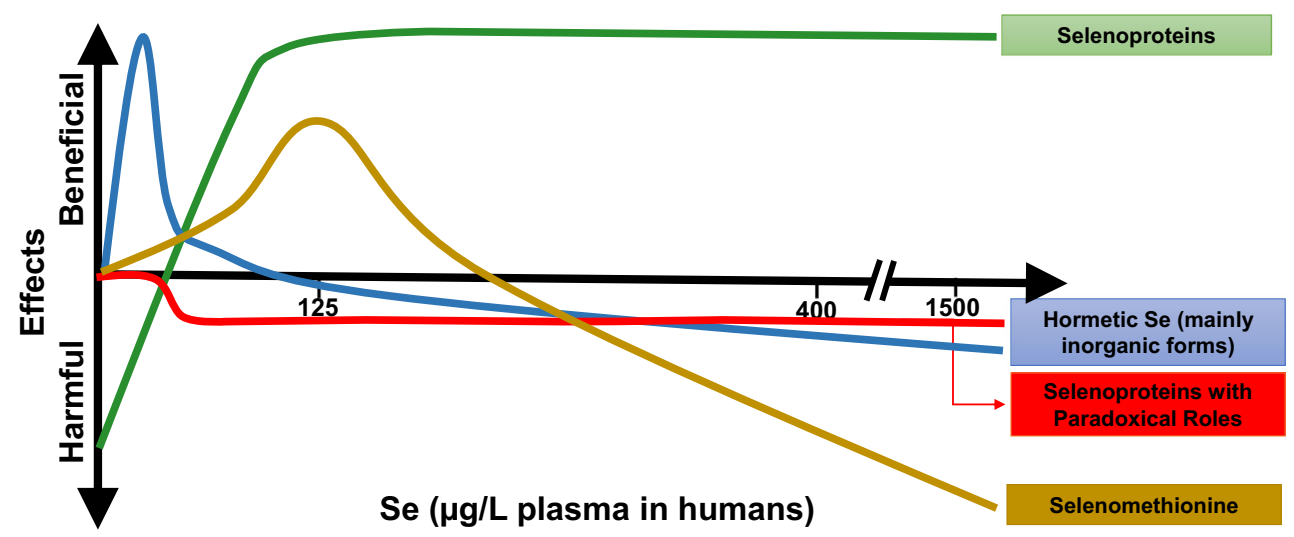

Fig. 3 Beneficial and harmful effects of Se depending on dose and form (speciation). It is proposed that Se has hermetic effects, shown by the blue line [43]. Selenoproteins with beneficial health effects are shown as a green line, while selenoproteins with paradoxical roles that appear to have harmful effects are shown as a red line [43-45]. As Se intake increases, particularly if the main source is SeMet, SeMet will increasingly replace methionine in body proteins, where its turnover is exceptionally slow; SeMet has toxic effects that are explained in the text (figure created with assistance from Wen-Hsing Cheng, Mississippi State University, Mississippi State, USA) 
generation of selenoproteins or excreted, and would not build up in the body in the same way as SeMet [48].

4. About one quarter of all bacteria express selenoproteins and therefore require Se for optimal growth [49]. Gut microorganisms are sensitive to trace elements and some require Se for normal metabolic functions [49]. In metabolizing Se, they produce volatile methylated Se compounds, such as dimethyldiselenide from SeMet, in the intestinal tract that are excreted, thus protecting the host from toxicity due to high Se exposure [50]. It is possible that exposure to high Se intake from birth or from a very young age alters the composition of the gut microbiota in such a way that excess Se is more readily excreted. Of course, this use of Se by microorganisms decreases the availability of $\mathrm{Se}$ for the expression of host selenoproteins, resulting in increased requirement of the host for Se [49].

\section{Conclusion}

The concept of the U-shaped relationship between Se intake/ status and its health effects, while useful, is clearly somewhat simplistic. We need a better understanding of the subtle factors outlined above that affect the Se requirements for optimal health, which vary by population and, indeed, between individuals. It would be interesting, for instance, to investigate whether the gut microbiome differs significantly in high vs. moderate consumers of Se. Se will still keep us guessing for some time to come.

\section{Compliance with ethical standards}

Conflict of interest As sole author, I declare that I have no conflict of interest.

Open Access This article is distributed under the terms of the Creative Commons Attribution 4.0 International License (http:// creativecommons.org/licenses/by/4.0/), which permits unrestricted use, distribution, and reproduction in any medium, provided you give appropriate credit to the original author(s) and the source, provide a link to the Creative Commons license, and indicate if changes were made.

\section{References}

1. Rayman MP (2005) Selenium in cancer prevention: a review of the evidence and mechanism of action. Proc Nutr Soc 64(4):527-542

2. Johnson CC, Fordyce FM, Rayman MP (2010) Symposium on 'geographical and geological influences on nutrition': factors controlling the distribution of selenium in the environment and their impact on health and nutrition. Proc Nutr Soc 69(1):119-132. https://doi.org/10.1017/s0029665109991807
3. Rayman MP (2012) Selenium and human health. Lancet 379(9822):1256-1268. https://doi.org/10.1016/s0140-6736(11) 61452-9

4. Xiong YM, Mo XY, Zou XZ et al (2010) Association study between polymorphisms in selenoprotein genes and susceptibility to Kashin-beck disease. Osteoarthr Cartil 18(6):817-824. https://doi. org/10.1016/j.joca.2010.02.004

5. Beck MA, Handy J, Levander OA (2004) Host nutritional status: the neglected virulence factor. Trends Microbiol 12(9):417-423. https://doi.org/10.1016/j.tim.2004.07.007

6. Bleys J, Navas-Acien A, Guallar E (2008) Serum selenium levels and all-cause, cancer, and cardiovascular mortality among us adults. Arch Intern Med 168(4):404-410. https://doi.org/10.1001/ archinternmed.2007.74

7. Avery JC, Hoffmann PR (2018) Selenium, selenoproteins, and immunity. Nutrients 10(9). https://doi.org/10.3390/nu10091203

8. Wu Q, Rayman MP, Lv H et al (2015) Low population selenium status is associated with increased prevalence of thyroid disease. J Clin Endocrinol Metab 100(11):4037-4047. https://doi.org/10. 1210/jc.2015-2222

9. Berr C, Balansard B, Arnaud J et al (2000) Cognitive decline is associated with systemic oxidative stress: the eva study. Etude du vieillissement arteriel. J Am Geriatr Soc 48(10):1285-1291

10. Akbaraly TN, Hininger-Favier I, Carriere I et al (2007) Plasma selenium over time and cognitive decline in the elderly. Epidemiology 18(1):52-58. https://doi.org/10.1097/01.ede. 0000248202.83695.4e

11. Gao S, Jin Y, Hall KS et al (2007) Selenium level and cognitive function in rural elderly chinese. Am J Epidemiol 165(8):955-965. https://doi.org/10.1093/aje/kwk073

12. Rayman MP, Stranges S (2013) Epidemiology of selenium and type 2 diabetes: can we make sense of it? Free Radic Biol Med 65:15571564. https://doi.org/10.1016/j.freeradbiomed.2013.04.003

13. Duffield-Lillico AJ, Reid ME, Turnbull BW et al (2002) Baseline characteristics and the effect of selenium supplementation on cancer incidence in a randomized clinical trial: a summary report of the nutritional prevention of cancer trial. Cancer Epidemiol Biomark Prev 11(7):630-639

14. Hughes DJ, Fedirko V, Jenab M et al (2015) Selenium status is associated with colorectal cancer risk in the european prospective investigation of cancer and nutrition cohort. Int J Cancer 136(5): 1149-1161. https://doi.org/10.1002/ijc.29071

15. Campa A, Baum MK, Bussmann H et al (2017) The effect of micronutrient supplementation on active tb incidence early in hiv infection in Botswana. Nutr Diet Suppl 2017(9):37-45. https://doi. org/10.2147/nds.s123545

16. Baum MK, Campa A, Lai S et al (2013) Effect of micronutrient supplementation on disease progression in asymptomatic, antiretroviral-naive, hiv-infected adults in Botswana: a randomized clinical trial. Jama 310(20):2154-2163. https://doi.org/10.1001/jama.2013. 280923

17. Morris JS, Crane SB (2013) Selenium toxicity from a misformulated dietary supplement, adverse health effects, and the temporal response in the nail biologic monitor. Nutrients 5(4): 1024-1057. https://doi.org/10.3390/nu5041024

18. Lippman SM, Klein EA, Goodman PJ et al (2009) Effect of selenium and vitamin e on risk of prostate cancer and other cancers: the selenium and vitamin e cancer prevention trial (select). Jama 301(1):39-51. https://doi.org/10.1001/jama.2008.864

19. Duffield-Lillico AJ, Slate EH, Reid ME et al (2003) Selenium supplementation and secondary prevention of nonmelanoma skin cancer in a randomized trial. J Natl Cancer Inst 95(19): 1477-1481

20. Rayman MP, Winther KH, Pastor-Barriuso R et al (2018) Effect of long-term selenium supplementation on mortality: results from a 
multiple-dose, randomised controlled trial. Free Radic Biol Med. https://doi.org/10.1016/j.freeradbiomed.2018.02.015

21. Stranges S, Marshall JR, Natarajan R et al (2007) Effects of longterm selenium supplementation on the incidence of type 2 diabetes: a randomized trial. Ann Intern Med 147(4):217-223

22. Kristal AR, Darke AK, Morris JS et al (2014) Baseline selenium status and effects of selenium and vitamin e supplementation on prostate cancer risk. J Natl Cancer Inst 106(3):djt456. https://doi. org/10.1093/jnci/djt456

23. Rayman MP, Duntas L (2019) Selenium deficiency and thyroid disease the thyroid and its diseases: a comprehensive guide for the clinician, 1st edn. Springer, Switzerland

24. White L, Romagne F, Muller E et al (2015) Genetic adaptation to levels of dietary selenium in recent human history. Mol Biol Evol 32(6):1507-1518. https://doi.org/10.1093/molbev/msv043

25. Ohta Y, Suzuki KT (2008) Methylation and demethylation of intermediates selenide and methylselenol in the metabolism of selenium. Toxicol Appl Pharmacol 226(2):169-177. https://doi.org/10.1016/j. taap.2007.09.011

26. Kuehnelt D, Engstrom K, Skroder H et al (2015) Selenium metabolism to the trimethylselenonium ion (tmse) varies markedly because of polymorphisms in the indolethylamine nmethyltransferase gene. Am J Clin Nutr 102(6):1406-1415. https://doi.org/10.3945/ajcn.115.114157

27. Liu Y, Zhang W, Zhao J et al (2018) Selenoprotein $\mathrm{p}$ as the major transporter for mercury in serum from methylmercury-poisoned rats. J Trace Elem Med Biol 50:589-595. https://doi.org/10.1016/ j.jtemb.2018.04.013

28. Suzuki KT, Sasakura C, Yoneda S (1998) Binding sites for the (hgse) complex on selenoprotein p. Biochim Biophys Acta 1429(1): 102-112

29. Manley SA, George GN, Pickering IJ et al (2006) The seleno bis(sglutathionyl) arsinium ion is assembled in erythrocyte lysate. Chem Res Toxicol 19(4):601-607. https://doi.org/10.1021/tx0503505

30. Sasakura C, Suzuki KT (1998) Biological interaction between transition metals (ag, cd and hg), selenide/sulfide and selenoprotein p. J Inorg Biochem 71(3-4):159-162

31. Jeppesen C, Valera B, Nielsen NO et al (2015) Association between whole blood mercury and glucose intolerance among adult inuit in Greenland. Environ Res 143(Pt A):192-197. https://doi.org/10. 1016/j.envres.2015.10.013

32. Achouba A, Dumas P, Ouellet N et al (2016) Plasma levels of selenium-containing proteins in inuit adults from nunavik. Environ Int 96:8-15. https://doi.org/10.1016/j.envint.2016.08.015

33. Hu XF, Eccles KM, Chan HM (2017) High selenium exposure lowers the odds ratios for hypertension, stroke, and myocardial infarction associated with mercury exposure among inuit in Canada. Environ Int 102:200-206. https://doi.org/10.1016/j. envint.2017.03.002

34. Hu XF, Sharin T, Chan HM (2017) Dietary and blood selenium are inversely associated with the prevalence of stroke among inuit in Canada. J Trace Elem Med Biol 44:322-330. https://doi.org/10. 1016/j.jtemb.2017.09.007

35. Passos CJ, Da Silva DS, Lemire M et al (2008) Daily mercury intake in fish-eating populations in the brazilian amazon. J Expo Sci Environ Epidemiol 18(1):76-87. https://doi.org/10.1038/sj.jes. 7500599
36. Lemire M, Philibert A, Fillion M et al (2012) No evidence of selenosis from a selenium-rich diet in the brazilian amazon. Environ Int 40:128-136. https://doi.org/10.1016/j.envint.2011.07. 005

37. IARC (2004) Some drinking-water disinfectants and contaminants, including arsenic. IARC Monogr Eval Carcinog Risks Hum 84:1477

38. Moxon AL (1938) The effect of arsenic on the toxicity of seleniferous grains. Science 88(2273):81. https://doi.org/10.1126/science. 88.2273.81

39. Pilsner JR, Hall MN, Liu X et al (2011) Associations of plasma selenium with arsenic and genomic methylation of leukocyte DNA in Bangladesh. Environ Health Perspect 119(1):113-118. https:// doi.org/10.1289/ehp.1001937

40. Matovic V, Buha A, Ethukic-Cosic D et al (2015) Insight into the oxidative stress induced by lead and/or cadmium in blood, liver and kidneys. Food Chem Toxicol 78:130-140. https://doi.org/10.1016/ j.fct.2015.02.011

41. Avissar N, Ornt DB, Yagil Y et al (1994) Human kidney proximal tubules are the main source of plasma glutathione peroxidase. Am J Phys 266(2 Pt 1):C367-C375. https://doi.org/10.1152/ajpcell.1994. 266.2.C367

42. Ruihua $Z$ et al (1991) Studies on the maximum safe Se intake of humans III. Certain antagonistic elements in blood and hair of inhabitants living in seleniferous area in Enshi. J Hygiene Res (3)

43. Zhang L, Zeng H, Cheng WH (2018) Beneficial and paradoxical roles of selenium at nutritional levels of intake in healthspan and longevity. Free Radic Biol Med 127:3-13. https://doi.org/10.1016/ j.freeradbiomed.2018.05.067

44. Zhou J, Huang K, Lei XG (2013) Selenium and diabetes-evidence from animal studies. Free Radic Biol Med 65:1548-1556. https:// doi.org/10.1016/j.freeradbiomed.2013.07.012

45. Misu H, Takamura T, Takayama $\mathrm{H}$ et al (2010) A liver-derived secretory protein, selenoprotein $\mathrm{p}$, causes insulin resistance. Cell Metab 12(5):483-495. https://doi.org/10.1016/j.cmet.2010.09.015

46. Rayman MP (2004) The use of high-selenium yeast to raise selenium status: how does it measure up? Br J Nutr 92(4):557-573

47. Swanson CA, Patterson BH, Levander OA et al (1991) Human [74se]selenomethionine metabolism: a kinetic model. Am J Clin Nutr 54(5):917-926

48. Rayman MP (2008) Food-chain selenium and human health: emphasis on intake. Br J Nutr 100(2):254-268. https://doi.org/10. 1017/s0007114508939830

49. Kasaikina MV, Kravtsova MA, Lee BC et al (2011) Dietary selenium affects host selenoproteome expression by influencing the gut microbiota. FASEB J 25(7):2492-2499. https://doi.org/10.1096/fj. 11-181990

50. Krittaphol W, McDowell A, Thomson CD et al (2011) Biotransformation of 1-selenomethionine and selenite in rat gut contents. Biol Trace Elem Res 139(2):188-196. https://doi.org/ 10.1007/s12011-010-8653-x

Publisher's note Springer Nature remains neutral with regard to jurisdictional claims in published maps and institutional affiliations. 